\title{
ANALYTIC APPROXIMATION OF INVASION WAVE AMPLITUDE PREDICTS SEVERITY OF INSECT OUTBREAKS*
}

\author{
JACOB P. DUNCAN ${ }^{\dagger}$ AND JAMES A. POWELL ${ }^{\dagger}$
}

\begin{abstract}
Outbreaks of phytophagous forest insects are largely driven by host demographics and spatial effects of dispersal. We develop a structured integrodifference equation (IDE) outbreak model that tracks the demographics of sedentary hosts under insect infestation pressure. The model is appropriate for a spectrum of pests attacking the later age classes of long-lived hosts, including mountain pine beetle (MPB), spruce budworm, and spruce beetle, which, among them are responsible for more forest damage than fire. The model generates a train of periodic waves of infestation. We approximate the IDE with a partial differential equation and search for traveling wave solutions. The resulting ordinary differential equation predicts the shape of an outbreak wave profile and peak infestation as functions of wavefront speed, which can be calculated analytically. This culminates in the derivation of an explicit approximation of invasion wave amplitude based on net reproductive rate of the infesting insect and its host searching efficiency. Results are compared with observations taken during a recent MPB outbreak in the northern US Rocky Mountains.
\end{abstract}

Key words. integrodifference equation, insect outbreak model, traveling waves, invasion speed, outbreak severity

AMS subject classification. 92

DOI. $10.1137 / 15 \mathrm{M} 1042085$

1. Introduction. Two of the primary mechanisms responsible for the irruptive nature of outbreak insect population dynamics are host demographic structure and spatial effects of insect dispersal (e.g., Bjørnstad et al. [11] and Aukema et al. [7]). Forest pests, unlike animal diseases, often only impact mature individuals, and so consideration of host demographics is critical in any sort of outbreak modeling framework. In the case of bark beetles (mountain pine beetle Dendroctonus ponderosae and spruce beetle Dendroctonus rufipennis) this is because only trees of sufficient size have thick enough phloem to allow larval survival. In the case of defoliators (spruce budworm Choristoneura fumiferana) this is because the largest trees dominate the canopy, and therefore have a disproportionately larger fraction of available foliage.

There is, however, a clear analogy between waves of disease in animal populations (Anderson and May $[4,5]$ ) and waves of infestation in forests. There is also an extensive literature on infectious disease models with demographic structure (Hethcote [20, 21], Keeling [23], Riley [31]). Heavilin and Powell [18] and Heavilin, Powell, and Logan [19] built several mountain pine beetle (MPB) outbreak models in the spirit of the classic SIR (susceptible-infected-recovered) infectious disease model (Kermack and McKendrick [24]). Though class structured, these models fall short of capturing the long recovery period of a forest affected by an outbreak of, for instance, the tree killing MPB. In order to model the dynamics of an outbreak cycle with an extended recovery period and make realistic predictions of severity, it is necessary to describe host demographics using age-structured models.

${ }^{*}$ Received by the editors October 1, 2015; accepted for publication (in revised form) October 24, 2016; published electronically February 22, 2017.

http://www.siam.org/journals/siap/77-1/M104208.html

${ }^{\dagger}$ Department of Mathematics and Statistics, Utah State University, Logan, UT 84322-3900 (jacob.duncan@aggiemail.usu.edu, jim.powell@usu.edu). 
While the MPB outbreak models of Heavilin and Powell [18] are successful in capturing the advance of infestation through a forest of healthy trees by addressing beetle dispersal, their model generates only two waves of infected trees (moving in opposite directions) which do not persist indefinitely as would be consistent with historical observations (Bleiker et al. [12], Alfaro et al. [2], Axelson, Alfaro, and Hawkes [8]). Abramson et al. [1], who modeled the spread of the hantavirus infection in deer mice using a continuous SIR-type epidemiological model coupled with a diffusion term for spatial dispersal of infectives, showed that sustained traveling wave trains of infection are possible. However, a continuous (e.g., differential equation) model is not appropriate for insect infestations in temperate climates on account of the discrete nature of insect life cycles. In particular, the MPB typically completes one generation per year which necessitates discrete-time modeling with difference equations.

The speed of an invading wave of infestation plays a critical role in determining the severity and duration of a local outbreak. The problem of finding the limiting velocity of wavefronts generated by reaction-diffusion equations dates back to Kolmogorov, Petrovsky, and Piskunov [25] and Fisher [17] who showed that, for particular types of reaction terms, this speed is twice the square root of the product of the diffusivity and intrinsic population growth rate of the invasive agent. Ben-Jacob et al. [9] addressed the question of how fast the front of a propagating disturbance (generated by a nonlinear diffusion equation) moves into an unstable, homogeneous region by viewing the wave speed as a damping constant for particle motion in a potential. They showed that the natural propagation speed corresponds to the critical-damping value. More generally, Aronson and Weinberger [6] rigorously showed that disturbances with compact support propagate with asymptotic speed equal to the minimal nonoscillatory wave speed.

There is a substantial amount of literature pertaining to the existence of traveling waves generated by disease outbreak models (for example, Ruan and Xiao [33]). However, methods for predicting the impact of a propagating wave on hosts are few. Sherratt $[35,36]$ derived explicit predictions of the amplitude and speed of periodic (invasion) wave trains generated by oscillatory reaction-diffusion equations. On the other hand, Duncan et al. [15] developed analytic methods for approximating the severity of an outbreak generated by an age-structured host demographic model, but which did not account for spatial structure induced by dispersal. Analytic techniques for predicting the severity of traveling periodic invasion waves arising from discretetime structured demographic models conjoined with dispersal components have not yet been developed.

In this paper, we analyze a sedentary host demographic model with outbreak insect dispersal motivated by the lodgepole-pine-tree-MPB interaction. The model is appropriate for a spectrum of pests attacking the later age classes of long-lived hosts, including MPB, spruce budworm, and spruce beetle, which, among them, are responsible for more forest damage than fire (Wood [38], Romme, Knight, and Yavitt [32], Logan, Régnière, and Powell [28]). The model consists of a system of integrodifference equations (IDE) that track the densities of pine trees infested by MPBs, healthy uninfested trees, and age classes of juvenile trees which are not susceptible to MPB attack due to insufficiently thick phloem layer for larval development. The age-structured demographic model is coupled with a Gaussian redistribution kernel to emulate (female) MPB dispersal each summer in their search for new susceptible host trees. Redistributing beetles across a landscape in which previously infested trees may not be reinfested for 50-100 years (via mortality and seedling regrowth to susceptible size) generates a train of sustained solitary waves of infestation that move through a 
forest with constant speed. At a fixed location in the forest, passing waves manifest temporally as periodic outbreaks much like those in typical stands with endemic MPB infestation (Alfaro et al. [2], Axelson, Alfaro, and Hawkes [8]).

We use a Wentzel-Kramers-Brillouin (WKB) (Dingle [14]) approximation in conjunction with the method of steepest descent (Erdelyi [16]) to evaluate the convolution integral in the IDE for infested trees, converting the difference equation into a second order nonlinear partial differential equation (PDE). The traveling wave ordinary differential equation (ODE) has a unique potential structure that allows determination of wave shape and amplitude (or peak), which we take as our measure of outbreak severity, in terms of tail behavior and wave speed. By linearizing the IDE, we calculate the rate of invasion, finding an explicit formula for predicting outbreak severity based on pest growth rate and searching efficiency. Results are compared with simulations as well as with observations taken during a recent MPB outbreak in central Idaho.

\section{Outbreak model.}

2.1. Host demographics. We analyze the age-structured host tree (lodgepole pine, Pinus contorta) MPB outbreak model developed by Duncan et al. [15],

$$
\begin{aligned}
j_{1, n+1}(x) & =(1-s) J_{n}(x)+I_{n-2}(x), \\
j_{k+1, n+1}(x) & =s j_{k, n}(x), \quad k=1, \ldots, K-1, \\
I_{n+1}(x) & =R I_{n}(x) e^{-\beta\left(T-S_{n}(x)+I_{n}(x)-s j_{K, n}(x)\right)}, \\
S_{n+1}(x) & =S_{n}(x)-I_{n}(x)+s j_{K, n}(x) .
\end{aligned}
$$

The difference equation model tracks population densities of susceptible trees (all adult trees older than maturation age $K$ when phloem thickness is sufficient to support development of an MPB brood), denoted $S_{n}(x)$, infested trees $I_{n}(x)$, and age classes of (nonsusceptible) juvenile trees $J_{n}(x)$ each year, $n$, at (one-dimensional) spatial location $x$. The density of $k$ year old juveniles in year $n$ is denoted by $j_{k, n}(x)$ while the total density of juveniles from all $K$ age classes is given by $J_{n}(x)=\sum_{k=1}^{K} j_{k, n}$. Juvenile survivorship, $s$, is assumed constant across age classes (2). Since natural mortality of mature lodgepole pines is negligible compared to infestation mortality during a major epidemic (Schmid and Amman [34]), the model assumes that susceptible trees are only killed by MPB infestation. This assumption leads to a slight overestimate in outbreak severity which we will take as a worst case scenario approximation.

Some species of conifers have evolved significant defensive responses to bark beetle attacks such as the secretion of resin to impede a beetle's ability to bore into a host tree (Amman and Cole [3]). It is necessary for MPBs to mass attack a tree to successfully overcome these antipredator adaptations (Berryman et al. [10]). Successful infestation kills the host tree within a year and lodgepole pines lose nearly all their needles roughly two years after infestation. Since lodgepole pine trees are shade intolerant, seeds only germinate in gaps in the forest floor left by dead trees. Therefore, the snag classes, $I_{n-1}$ and $I_{n-2}$, must be included in the model because they still shade the forest floor, delaying initiation of juvenile recruitment (1). In the case of defoliators, a similar dynamic occurs since generally two years of defoliation are required to kill an adult tree, after which its shade footprint is available for juveniles. Trees that survive to age $K+1$ years are large enough for successful MPB infestation and, thus, graduate (mature) into the class of susceptibles. In year $n+1$, susceptible tree density will be 
its previous value, minus the number of trees that became infested, plus the number of $K$ year old juveniles that survived the year, hence (4).

The exponential factor in (3) represents the probability of infesting beetles encountering new susceptible trees in a Poisson search process with failure rate $\beta$, i.e., host searching inefficiency (see Powell and Bentz [29] for details). Here $T-S_{n}(x)+$ $I_{n}(x)-s j_{K, n}(x)$ represents the density of nonsusceptible trees under the assumption that forest floor space is finite with host tree stand carrying capacity, represented by $T$. The number of new infestations per infested tree, which can be viewed as the net reproductive rate of MPBs, is denoted by $R$.

A detailed development of this model, including fixed point stability analysis and outbreak severity approximation, can be found in Duncan et al. [15]. The salient results are that the infestation-free state loses stability for $R \geq 1$; an endemic fixed point comes into existence and is stable for values of $R$ larger than 1 but less than some critical threshold, above which the system settles into large oscillations corresponding to periodic outbreaks. We note that parameter values must satisfy $T>\frac{2 \ln R}{\beta}$ (derived from the outbreak severity prediction in Duncan et al. [15]) to preclude the model from predicting infested tree densities that exceed the carrying capacity of the forest. For realistic parameters range estimates this condition is always true. Table 1 summarizes the model variables, parameters, units, and estimated nominal values. Figure 1 diagrams the evolution of host tree demographics over the course of one MPB generation (1 year) as well as the entire life history of a host.

TABLE 1

MPB outbreak model variables and parameters. Powell and Bentz [29, 30] estimated $R, \beta, \gamma, \alpha$, and $\sigma$ from data taken during a recent outbreak of MPB in the Sawtooth National Recreation Area (SNRA), Idaho. Total host density $T$ is estimated for the SNRA in Crabb, Powell, and Bentz [13]. Estimates for $K$ and $d$ were determined using reference values consistent with field observation. See Duncan et al. [15] for details.

\begin{tabular}{|c|c|c|}
\hline Variables & Description & Units \\
\hline$S_{n}(x)$ & $\begin{array}{l}\text { Susceptible tree density in year } n \text { at } \\
\text { location } x\end{array}$ & stems/ha \\
\hline$I_{n}(x)$ & $\begin{array}{l}\text { Infested tree density in year } n \text { at loca- } \\
\text { tion } x\end{array}$ & stems/ha \\
\hline$j_{k, n}(x)$ & $\begin{array}{l}\text { Juvenile tree density of the } k \text { th age } \\
\text { class in year } n \text { at location } x\end{array}$ & stems/ha \\
\hline$J_{n}(x)$ & Total juvenile tree density & stems/ha \\
\hline$C_{n}(x)$ & $\begin{array}{l}\text { Density of beetles colonizing trees at } \\
\text { the end of the dispersal season in year } \\
n\end{array}$ & MPB/ha \\
\hline Parameters & Description & Estimated Values/Units \\
\hline$T$ & Total number of trees per hectare & $T=390$ stems $/$ ha \\
\hline$K$ & Number of juvenile age classes & $K=80$ age classes \\
\hline$d$ & Natural mortality rate for juveniles & $d=0.01$ per year \\
\hline$s=1-d$ & Natural juvenile survivorship & $s=0.99$ per year \\
\hline$\beta$ & Failure rate in MPB search process & $\beta=0.011 \mathrm{ha} / \mathrm{stem}$ \\
\hline$R=\gamma \alpha$ & Net reproductive rate of MPB & $R=2.16$ per year \\
\hline$\sigma$ & $\begin{array}{l}\text { Standard deviation of Gaussian disper- } \\
\text { sal kernel }\end{array}$ & $\sigma=253.5 \mathrm{~m}$ \\
\hline Values to approximate & Description & Units \\
\hline$c^{*}$ & $\begin{array}{l}\text { Speed of an invading infestation wave- } \\
\text { front }\end{array}$ & $\mathrm{m} /$ year \\
\hline$\tau^{*}$ & $\begin{array}{l}\text { Shape parameter of an invading wave- } \\
\text { front }\end{array}$ & $1 / \mathrm{m}$ \\
\hline$I_{\max }$ & $\begin{array}{l}\text { Maximum MPB impact in a single year } \\
\text { throughout an outbreak }\end{array}$ & stems/ha \\
\hline
\end{tabular}

Copyright (c) by SIAM. Unauthorized reproduction of this article is prohibited. 
(a)

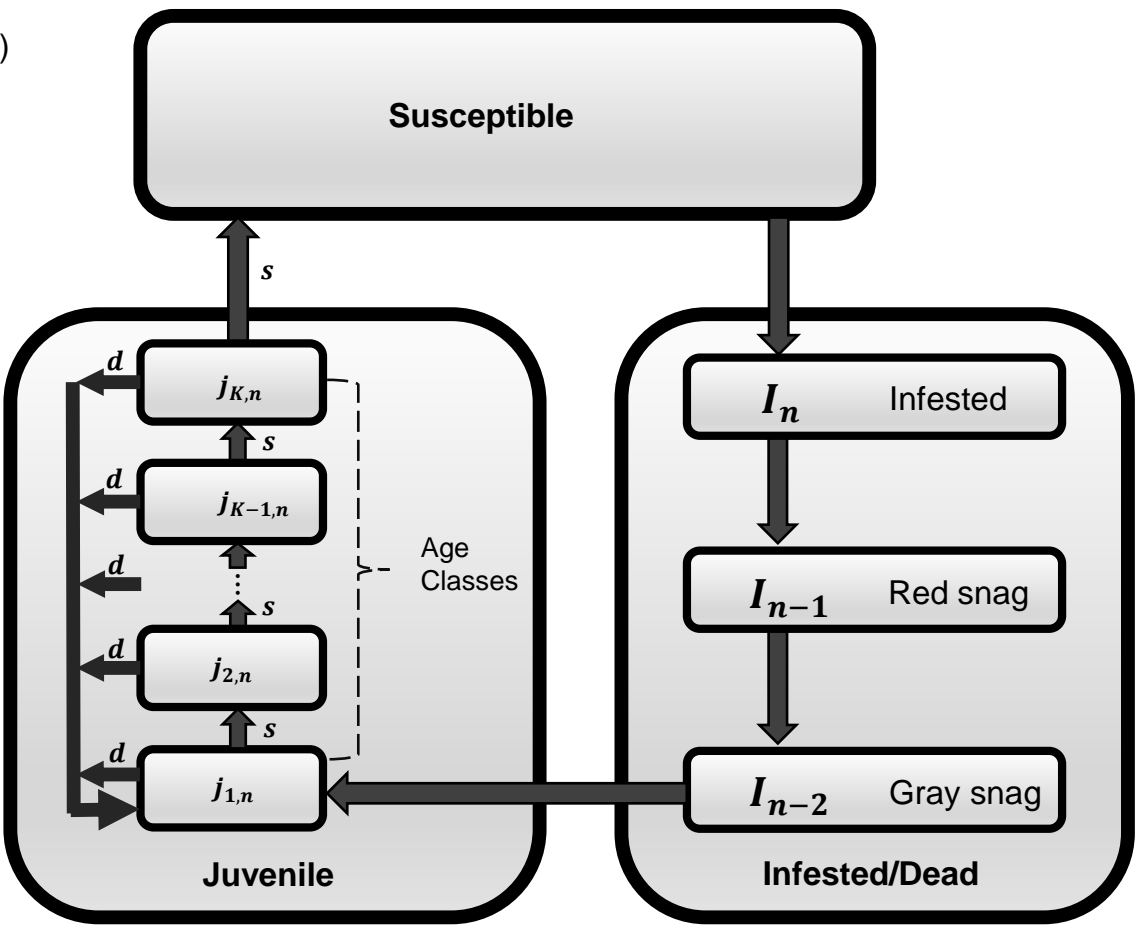

(b)

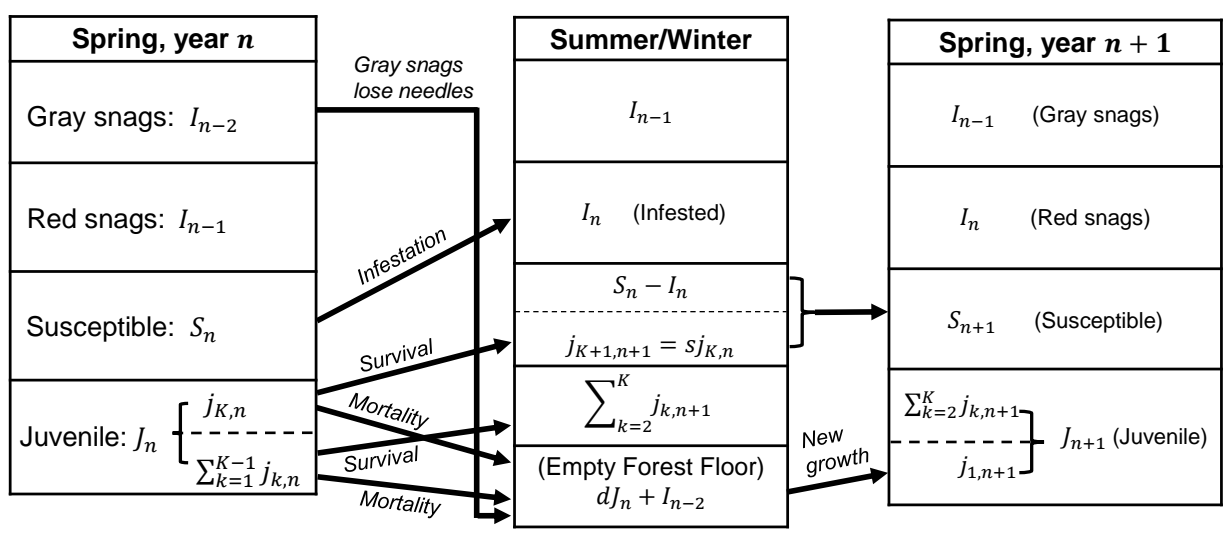

FIG. 1. (a) Host tree lifecycle diagram. Natural juvenile mortality, d, opens up forest floor space to new seedling growth. Likewise, infestation mortality translates to seedling growth (after a once infested tree spends two years as a snag). Juvenile age class survivorship $s=1-d$ is constant. Trees that survive to age $K+1$ years graduate (mature) into the class of susceptibles. (b) In spring, juvenile trees either die and leave empty spaces on the forest floor, or survive and graduate to the next age class. In summer, a susceptible tree either becomes infested or avoids infestation and remains in the class of susceptible trees. Over the winter, two year old snags lose all their needles creating a gap in the forest floor. In spring, seedlings sprout in any open forest floor space created by natural juvenile mortality or infestation mortality. 
2.2. Insect dispersal. MPBs disperse in late summer in search of new hosts after emerging from previously infested trees, which have been killed by the burrowing larvae and associated fungal pathogens. Perpendicular to a (large) wave of infestation, the spread of infested trees can be viewed as a one-dimensional transect, and so we track densities in units of stems/ha even though dispersal is one dimensional along the transect, following Kot, Lewis, and van den Dreissch [26]. Powell and Bentz [30] use observations of infestation progress from year to year to demonstrate that MPBs disperse diffusively according to the ecological diffusion equation, in which beetle motility depends on host density. Here we assume that the density of lodgepole hosts is constant, and therefore we model beetle dispersal on a continuous one-dimensional habitat using the diffusion equation,

$$
\begin{aligned}
\frac{\partial P}{\partial t} & =D \frac{\partial^{2} P}{\partial x^{2}},-\infty<x<\infty, t>0, \\
P(x, 0) & =\alpha I_{n}(x),
\end{aligned}
$$

where $D$ is the (constant) rate at which beetles disperse, and $\alpha$ is the mean number of MPBs that emerge from an infested tree. Powell and Bentz [30] estimate mean MPB emergence at $\alpha=2043 \mathrm{MPBs} / \mathrm{stem}$ using infestation data taken during a recent outbreak in the SNRA of central Idaho.

To incorporate beetle dispersal in the demographic model, we rewrite (3) as

$$
I_{n+1}(x)=\gamma C_{n}(x) e^{-\beta\left(T-S_{n}(x)+I_{n}(x)-s j_{K, n}(x)\right)},
$$

where $C_{n}(x)$ denotes the density of colonizing MPBs at the end of the previous summer and $\gamma$ represents the number of trees that can be colonized per attacking beetle. An estimated 250 attacking beetles per tree are required to successfully overcome a host's defensive mechanisms, and subsequent attacks fill up trees at a rate of $698 \mathrm{MPBs} /$ host according to Powell and Bentz [30]. Since we are constructing an outbreak model wherein beetle populations are generally well above the attack threshold (250 MPB/stem), we can combine the beetle-to-infested tree conversion rate $\left(\frac{1}{698}\right.$ stems $\left./ \mathrm{MPB}\right)$ with the attack threshold to get an estimate of $\gamma=\frac{1}{948}$, where $948=250+698$.

When susceptible host density is high, the number of new infestations is simply proportional to the number of attacking beetles which we compute by the convolution,

$$
C_{n}(x)=\int_{-\infty}^{\infty} k(x-y) \alpha I_{n}(y) d y
$$

where $k$ is the fundamental solution of (5) (with $t=1$ ),

$$
k(x)=\frac{1}{\sqrt{2 \pi} \sigma} e^{\frac{-x^{2}}{2 \sigma^{2}}} .
$$

Here we have the relation between dispersal rate and variance of the Gaussian distribution, $\sigma^{2}=2 D t$, where $t=1$ since individual MPBs only disperse for a single day before finding new hosts (Logan and Powell [27], Powell and Bentz [29]). Combining the demographic and dispersal models, we write (6) as the IDE

$$
I_{n+1}(x)=R e^{-\beta\left(T-S_{n}(x)+I_{n}(x)-s j_{K, n}(x)\right)} \int_{-\infty}^{\infty} k(x-y) I_{n}(y) d y,
$$

where $R=\gamma \alpha$ represents the yearly MPB population growth rate.

For simulations used to obtain invasion speeds and severities, we initialized with a small infestation at the origin and integration in (7) was performed using the fast 
Fourier transform on a domain (centered about the origin) large enough to preclude the initial traveling waves from reaching the boundary. The number of partition points in the domain was chosen so that $\Delta x$ never exceeded 0.1 . The model was iterated until the transient dynamics of the initial waves had significantly dissipated (usually after 20 generations) before diagnosing (asymptotic) wave speed and peak. We calculated invasion speed by taking the average distance traveled by the (rightmoving) leading wave's peak per year. The leading wave's amplitude was found by taking the maximum value of $I_{n}(x)$ over the right half of the spatial domain since the leading wave's peak is always higher than the subsequent train of periodic waves.

A localized perturbation (at the origin) of the unstable trivial steady state (no infestation) generates a train of solitary infestation waves that propagate through an undisturbed medium of host trees (Figure 2). The initial waves of infestation traveling through space correspond to outbreaks in time for a particular point on the landscape. The subsequent train of periodic waves generated after the initial leading wave have smaller peaks and less steep edges since they are moving into a region with less than ideal conditions, i.e., with sizable densities of nonsusceptible trees whereas the initial wave invades area saturated with susceptible hosts. In what follows, we estimate the natural propagation speed of the leading wave, which is in turn used to construct an approximation of outbreak severity as measured by wavefront amplitude (peak infestation).

3. Predicting the speed of an invading wavefront. To predict the speed of the leading wave generated by an initial pulse of infested trees, we first formulate the model in terms of matrices, linearize, and assume wave tails are exponential in form. In a moving frame of reference near the front of the invasion, a point on next generation's front is a horizontal translation and a vertical multiple of some point on the current front. Thus, using dominant eigenvalues of the linearized model to estimate wave growth behavior, we can predict the invasion speed by choosing the speed corresponding to neutral stability in the frame of reference traveling with the wave (Jones, Kapitula, and Powell [22]).

3.1. Asymptotic traveling wave speed. Our model can be written in matrix form as

$$
\mathbf{Y}_{n+1}(x)=\int_{-\infty}^{\infty} \mathbf{B}_{\mathbf{Y}}(x, y) \mathbf{Y}_{n}(y) d y
$$

where

$$
\mathbf{Y}_{n}=\left[\begin{array}{llllllll}
j_{1, n} & j_{2, n} & \cdots & j_{K, n} & S_{n} & I_{n} & I_{n-1} & I_{n-2}
\end{array}\right]^{\mathrm{T}}
$$

denotes the population densities of all classes of trees, and the matrix

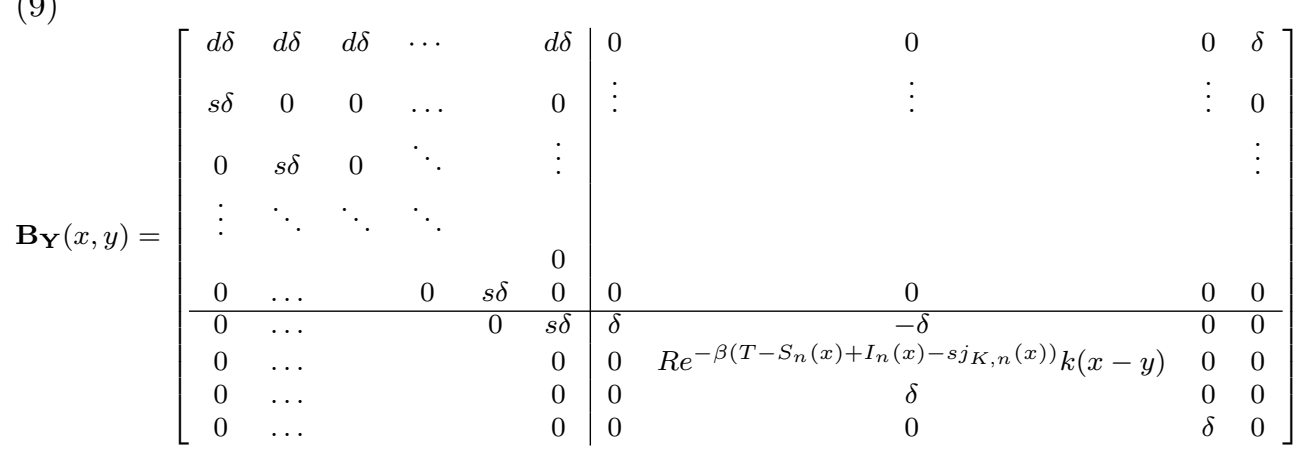




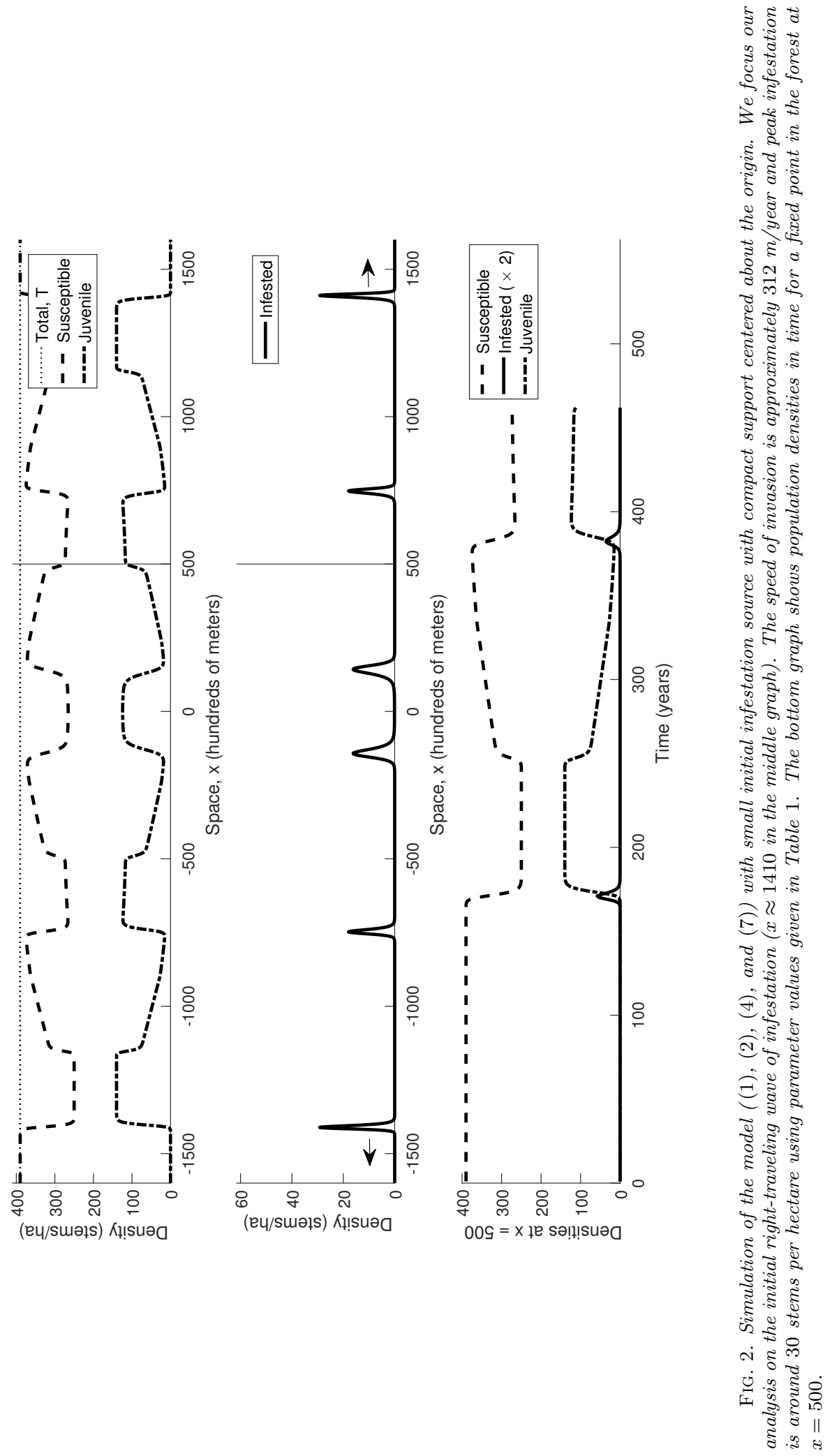


encapsulates density-dependent host population growth and insect dispersal. Natural juvenile mortality rate is denoted by $d=1-s$, and we use the Dirac delta function $\delta=\delta(x-y)$ to model the absence of dispersal in transitions between any two stages other than from $I_{n}$ to $I_{n+1}$. In transitioning from $I_{n}$ to $I_{n+1}$, infested trees beget more infested trees through beetle dispersal (via the Gaussian kernel, $k$ ) and colonization. Matrix $\mathbf{B}$ is partitioned with the upper-left $K \times K$ block corresponding to the juvenile age classes.

To approximate the speed of traveling waves generated by the model, we linearize around the trivial steady state,

$$
\tilde{\mathbf{Y}}=\left[\begin{array}{lllllll}
0 & \ldots & 0 & T & 0 & 0 & 0
\end{array}\right]^{\mathrm{T}},
$$

assuming that before an MPB invasion moves in, the forest consists of susceptible trees and negligible densities of juvenile and infested trees. To calculate the speed of waves generated by the linearized model, let $\mathbf{A}=\mathbf{B}_{\tilde{\mathbf{Y}}}$ be the Fréchet derivative of $\mathbf{B}$ at $\tilde{\mathbf{Y}}$. By the linear conjecture of van den Bosch, Metz, and Diekmann [37], the speed of an invasion wave generated by a nonlinear model can be approximated by its linearization at low invasive population densities. The linearized model takes the form of a convolution, which can be written as

$$
\mathbf{Y}_{n+1}(x)=\int_{-\infty}^{\infty} \mathbf{A}(y) \mathbf{Y}_{n}(x-y) d y
$$

where

$$
\mathbf{A}(y)=\left[\begin{array}{cccccc|cccc}
d \delta & d \delta & d \delta & \cdots & & d \delta & 0 & 0 & 0 & \delta \\
s \delta & 0 & 0 & \ldots & & 0 & \vdots & \vdots & \vdots & 0 \\
0 & s \delta & 0 & \ddots & & \vdots & & & & \vdots \\
\vdots & \ddots & \ddots & \ddots & & & & & & \\
& & & & & 0 & & & & \\
0 & \ldots & & 0 & s \delta & 0 & 0 & 0 & 0 & 0 \\
\hline 0 & \ldots & & & 0 & s \delta & \delta & -\delta & 0 & 0 \\
0 & \ldots & & & & 0 & 0 & R k(y) & 0 & 0 \\
0 & \ldots & & & & 0 & 0 & \delta & 0 & 0 \\
0 & \cdots & & & & 0 & 0 & 0 & \delta & 0
\end{array}\right] .
$$

In a moving frame of reference with unknown speed $c>0$ near the invasion front, we assume

$$
\mathbf{Y}_{n+1}(x)=\mathbf{Y}_{n}(x-c) e^{\varepsilon},
$$

where $\varepsilon$ is an unknown wave growth parameter and $\mathbf{Y}$ is the solution of the linearized model (11). That is, we imagine that the wave is translating to the right with speed $c$ and growing vertically by a factor of $e^{\varepsilon}$ each year with respect to the observer's frame of reference (Figure 3(a)).

Suppose the leading edge of the wavefront is of the form

$$
\mathbf{Y}_{n}(x)=e^{-\tau x} \mathbf{v},
$$

where $\mathbf{v}$ gives the (constant) relative abundance of each tree class in the traveling wave and $\tau$ determines the shape of the advancing edge of the wave. Then the linearized model (11) becomes

$$
e^{\tau c+\varepsilon} \mathbf{v}=\mathbf{H}(\tau) \mathbf{v}
$$



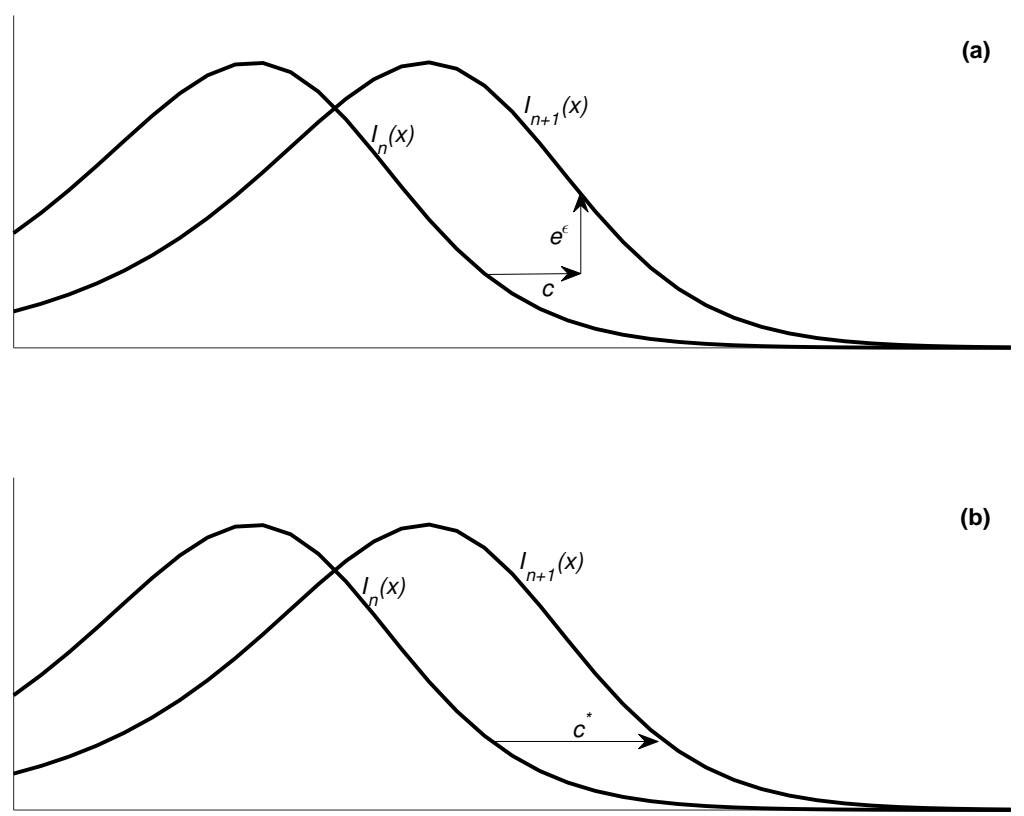

FIG. 3. (a) In a moving frame of reference with speed $c$ near the front of the invasion, a point on next generation's front is a horizontal translation, $c$, and a vertical multiple, $e^{\varepsilon}$, of some point on the current front. (b) We predict the speed of the invasion by choosing the frame of reference speed, $c^{*}$, that corresponds to a vertical multiple of unity $(\varepsilon=0)$.

where $\mathbf{H}(\tau) \equiv \int_{-\infty}^{\infty} \mathbf{A}(y) e^{\tau y} d y$. Since the behavior of $e^{\tau c+\varepsilon} \mathbf{v}$ is dictated by the dominant eigenvalue of $\mathbf{H}(\tau)$, denoted $\rho(\tau)$, we have

$$
e^{\tau c+\varepsilon} \mathbf{v} \sim \rho(\tau) \mathbf{v}
$$

and thus,

$$
\varepsilon=-\tau c+\ln [\rho(\tau)] .
$$

Furthermore, since the long-term behavior of wave growth is controlled by the maximum value of $\varepsilon$, setting $\frac{d \varepsilon}{d \tau}=0$ using (14) gives

$$
c=\frac{\rho^{\prime}(\tau)}{\rho(\tau)} .
$$

Since $\varepsilon=0$ in a frame of reference moving with the wavefront, (14) becomes

$$
c=\frac{1}{\tau} \ln \rho(\tau)
$$

(Figure 3(b)). From (15) and (16), we have $\frac{\rho^{\prime}(\tau)}{\rho(\tau)}=\frac{1}{\tau} \ln \rho(\tau)$, which is the condition that arises when minimizing $c$ in (16) (and therefore maximizing $\varepsilon$ according to (14)). The predicted asymptotic wave speed is thus

$$
c^{*}=\min _{0<\tau}\left(\frac{1}{\tau} \ln \rho(\tau)\right) .
$$

Copyright $@$ by SIAM. Unauthorized reproduction of this article is prohibited. 
3.2. Speed of model-generated invasion wave. To predict the speed of the wavefront, we require the spectral radius of

$$
\mathbf{H}(\tau)=\left[\begin{array}{ccccccc|ccc}
d & d & d & \cdots & d & 0 & 0 & 0 & 1 \\
s & 0 & 0 & \ldots & 0 & \vdots & \vdots & \vdots & 0 \\
0 & s & 0 & \ddots & \vdots & & & \vdots \\
\vdots & \ddots & \ddots & \ddots & & & & & \\
& & & & & 0 & & & & \\
0 & \ldots & & 0 & s & 0 & 0 & 0 & 0 & 0 \\
0 & \ldots & & & 0 & s & 1 & -1 & 0 & 0 \\
\hline 0 & \ldots & & & 0 & 0 & R e^{\frac{\sigma^{2}}{2}} \tau^{2} & 0 & 0 \\
0 & \ldots & & & 0 & 0 & 1 & 0 & 0 \\
0 & \ldots & & & 0 & 0 & 0 & 1 & 0
\end{array}\right],
$$

which is block upper triangular and hence its spectrum is the union of the spectra of the diagonal blocks. Let $\mathbf{H}_{1}$ and $\mathbf{H}_{2}$ denote the upper-left and lower-right blocks, respectively. By the Gershgorin circle theorem with respect to the columns of $\mathbf{H}_{1}$, the spectral radius of $\mathbf{H}_{1}$ is no more than 1 . Since the spectral radius of $\mathbf{H}_{2}(\tau)$ is $R e^{\frac{\sigma^{2}}{2} \tau^{2}}$, the largest eigenvalue of $\mathbf{H}(\tau)$ is $\rho(\tau)=R e^{\frac{\sigma^{2}}{2} \tau^{2}}$, provided $R>1$ (which is required for a growing population of pests).

Applying (17), we have an approximation of the speed of the invading wavefront,

$$
c^{*}=\sigma \sqrt{2 \ln R},
$$

where the minimizing value of the wave shape parameter is

$$
\tau^{*}=\frac{\sqrt{2 \ln R}}{\sigma} .
$$

Thus, we have closed-form approximations of the speed and shape of the right tail of the (right-traveling) invasion wave profile in terms of model parameters alone.

4. Invasion wave amplitude approximation. Our goal now is to approximate the amplitude of the invading wave as well as the shapes of the right and left tails in terms of wave speed, which is left as a free parameter to illuminate the unique structure of the traveling wave ODE potential. Using our approximation of asymptotic wave speed, $c^{*}$, we derive analytic predictions of wave amplitude and tail shapes explicitly in terms of pest growth rate and search efficiency. To begin, we derive an approximating PDE from the IDE for infested hosts (7).

4.1. Converting the IDE into a PDE. Since the tails of the invading wave appear to be exponential, we employ the WKB method whereby we assume the solution of the integral equation (7) is of the form

$$
I_{n}(x)=e^{u_{n}(x)}
$$

for some function $u_{n}(x) \in C^{2}$. Then the convolution $\left(k * I_{n}\right)(x)$ becomes

$$
k * e^{u_{n}}=\int_{-\infty}^{\infty} \frac{1}{\sqrt{2 \pi} \sigma} e^{h(y)} d y
$$


where

$$
h(y)=u_{n}(x-y)-\frac{y^{2}}{2 \sigma^{2}} .
$$

We approximate this integral using the method of steepest descent. Expanding $u_{n}$ in its Taylor series, we have

$$
h(y)=u_{n}(x)-u_{n}^{\prime}(x) y+\frac{u_{n}^{\prime \prime}(x)}{2 !} y^{2}-\cdots-\frac{y^{2}}{2 \sigma^{2}} .
$$

Ignoring higher order terms, the critical point of $h\left(\right.$ denoted $\left.y^{*}\right)$ satisfies

$$
h^{\prime}\left(y^{*}\right)=-u_{n}^{\prime}(x)+u_{n}^{\prime \prime}(x) y^{*}-\frac{y^{*}}{\sigma^{2}}=0,
$$

which implies

$$
y^{*}=-\sigma^{2} u_{n}^{\prime}(x) \frac{1}{1-\sigma^{2} u_{n}^{\prime \prime}(x)} .
$$

Expanding as a power series in $\sigma^{2}$, we have

$$
y^{*}=-\sigma^{2} u_{n}^{\prime}(x)\left(1+\sigma^{2} u_{n}^{\prime \prime}(x)+\sigma^{4}\left(u_{n}^{\prime \prime}(x)\right)^{2}+\cdots\right) .
$$

Now

$$
h\left(y^{*}\right)=u_{n}(x)+\frac{1}{2} \sigma^{2}\left(u_{n}^{\prime}(x)\right)^{2}+\mathcal{O}\left(\sigma^{4}\right),
$$

and at leading order,

$$
h^{\prime \prime}\left(y^{*}\right)=u_{n}^{\prime \prime}(x)-\frac{1}{\sigma^{2}} \approx-\frac{1}{\sigma^{2}},
$$

provided $\sigma^{2} \ll 1$. Thus, by the method of steepest descent,

$$
k * I_{n} \approx \frac{1}{\sqrt{2 \pi} \sigma} \sqrt{\frac{2 \pi}{\left|h^{\prime \prime}\left(y^{*}\right)\right|}} e^{h\left(y^{*}\right)}=e^{u_{n}(x)+\frac{1}{2} \sigma^{2}\left(u_{n}^{\prime}(x)\right)^{2}+\mathcal{O}\left(\sigma^{4}\right)} .
$$

We now move to a continuous setting by transforming (7) to an (approximately) equivalent PDE. Neglecting higher order terms in (22), we can rewrite (7) as

$$
e^{u_{n+1}}=R e^{-\beta\left(T-S_{n+1}\right)} e^{u_{n}+\frac{1}{2} \sigma^{2}\left(u_{n}^{\prime}\right)^{2}},
$$

and setting the index back one,

$$
e^{u_{n}}=R e^{-\beta\left(T-S_{n}\right)} e^{u_{n-1}+\frac{1}{2} \sigma^{2}\left(u_{n-1}^{\prime}\right)^{2}},
$$

where $u^{\prime}$ denotes differentiation with respect to $x$. Dividing (23) by (24) yields

$$
e^{u_{n+1}-u_{n}}=e^{u_{n}-u_{n-1}+\frac{1}{2} \sigma^{2}\left[\left(u_{n}^{\prime}\right)^{2}-\left(u_{n-1}^{\prime}\right)^{2}\right]} e^{\beta\left(S_{n+1}-S_{n}\right)} .
$$

Since $j_{K, n}$ is negligible in the leading edge of the wavefront, $S_{n+1}-S_{n}=-I_{n}+s j_{K, n} \approx$ $-e^{u_{n}}$ and, hence, after equating exponents in (25) and rearranging, we have

$$
u_{n+1}-2 u_{n}+u_{n-1}=\frac{1}{2} \sigma^{2}\left[\left(u_{n}^{\prime}\right)^{2}-\left(u_{n-1}^{\prime}\right)^{2}\right]-\beta e^{u_{n}} .
$$

Suppose $u_{n}(x)=w(t=n \Delta t, x)$ for some twice continuously differentiable function $w$ and time step $\Delta t=1$. Then (26) is approximately the second order nonlinear PDE,

$$
w_{t t}=\sigma^{2} w_{x} w_{x t}-\beta e^{w},
$$

upon Taylor expanding $u_{n-1}^{\prime}(x)=w_{x}((n-1) \Delta t, x)$.

Copyright $@$ ( ) by SIAM. Unauthorized reproduction of this article is prohibited. 
4.2. Traveling wave profile. We look for traveling wave solutions of (27) by assuming $w=f(z)$, where $z=x-$ ct. Substituting into (27),

$$
c^{2} f^{\prime \prime}=-c \sigma^{2} f^{\prime} f^{\prime \prime}-\beta e^{f}
$$

which, after multiplying by $f^{\prime}$, integrates to

$$
\frac{1}{2} c^{2}\left(f^{\prime}\right)^{2}+\frac{1}{3} c \sigma^{2}\left(f^{\prime}\right)^{3}+\beta e^{f}=C
$$

for some constant $C$. We may interpret (28) as the mechanical equation of motion for a particle with position $f$ and velocity $f^{\prime}$ (Ben-Jacob et al. [9]). Then $C$ can be viewed as the "energy" along trajectories where

$$
E_{1}\left(f^{\prime}\right) \equiv \frac{1}{2} c^{2}\left(f^{\prime}\right)^{2}+\frac{1}{3} c \sigma^{2}\left(f^{\prime}\right)^{3}
$$

represents the "energy of motion" and

$$
E_{2}(f) \equiv \beta e^{f}
$$

represents the "energy of position." Solutions of (28) are isoclines of $E_{1}+E_{2}$. The amplitude of the invading wave, $I_{\max }$, represents peak infestation which is the measure of outbreak severity we seek to estimate. Supposing $I_{n}(x)$ attains its maximum value, $I_{\max }$, at $x=0$ when $n=t=0$, we find $C=\beta I_{\max }$. At this point, $E_{1}$ is at a local minimum, $\left(E_{1}(0)=0\right)$ and $E_{2}\left(\ln I_{\max }\right)=\beta I_{\max }$ (Figure 4). The maximum value of $E_{1}$ generating bounded $f^{\prime}$ is $\frac{c^{4}}{6 \sigma^{4}}$ (imagining a particle rolling on the curve in Figure 4(b)), i.e., if $\frac{-c}{\sigma^{2}} \leq f^{\prime} \leq \frac{c}{2 \sigma^{2}}$, then $0 \leq E_{1} \leq \frac{c^{4}}{6 \sigma^{4}}$.

We require $I_{n}(x) \rightarrow 0$ as $x \rightarrow \pm \infty$ which forces $f(z) \rightarrow-\infty$ since $f=\ln I$. As $f \rightarrow-\infty,(28)$ becomes

$$
\frac{1}{2} c^{2}\left(f^{\prime}\right)^{2}+\frac{1}{3} c \sigma^{2}\left(f^{\prime}\right)^{3}=\beta I_{\max } .
$$

We choose the maximum (feasible) value of $E_{1}$, when $f^{\prime}=\frac{-c}{\sigma^{2}}$ or $f^{\prime}=\frac{c}{2 \sigma^{2}}$, in order to obtain a worst case scenario (i.e., largest possible peak) approximation of $I_{\max }$. From (29) we have

$$
I_{\max }=\frac{c^{4}}{6 \beta \sigma^{4}} .
$$

Figure 5 shows a phase diagram of (28) with the trajectory corresponding to the maximum value of $E_{1}$.

The horizontal asymptotes, $\frac{-c}{\sigma^{2}}$ and $\frac{c}{2 \sigma^{2}}$ of the maximum amplitude trajectory in Figure 5 represent the slopes of $f$ as $z \rightarrow \pm \infty$, respectively. For fixed $n, \ln \left(I_{n}(x)\right)=$ $\ln (I(x)) \approx f(x-c t)=f(z)$ for fixed $t$ and therefore,

$$
f^{\prime}(z)=\frac{I^{\prime}(x)}{I(x)} \rightarrow \frac{-c}{\sigma^{2}} \text { as } z \rightarrow+\infty
$$

and

$$
f^{\prime}(z)=\frac{I^{\prime}(x)}{I(x)} \rightarrow \frac{c}{2 \sigma^{2}} \text { as } z \rightarrow-\infty
$$

Copyright (C) by SIAM. Unauthorized reproduction of this article is prohibited. 


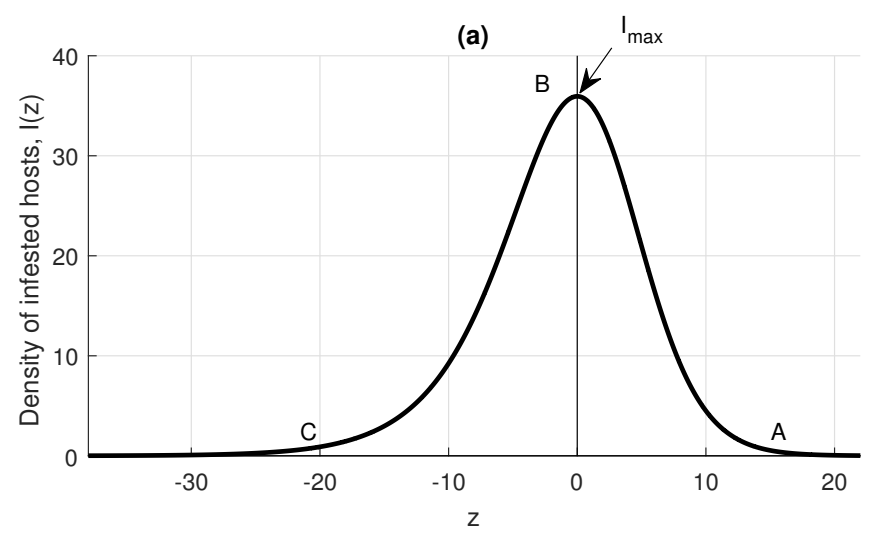

(b)

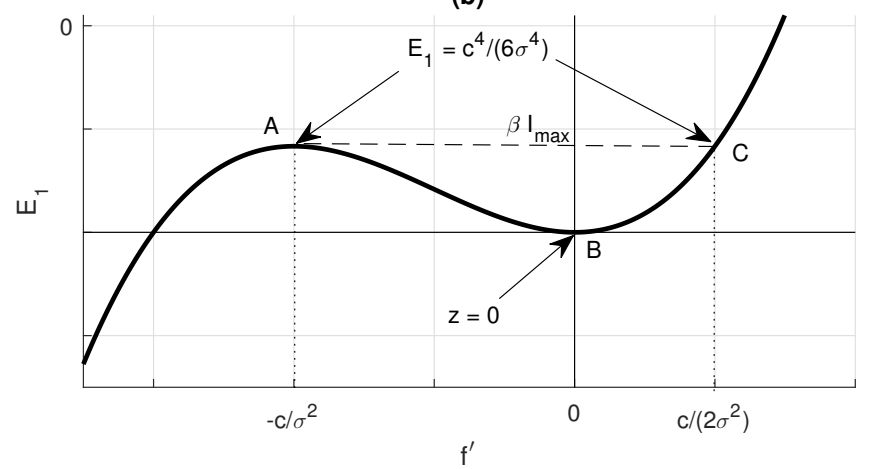

(c)

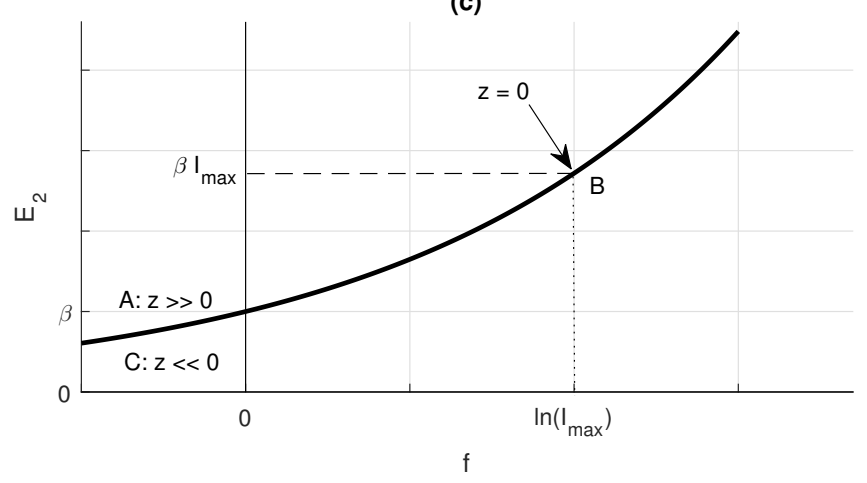

FIG. 4. The left-hand side of the traveling wave equation (28) contains the kinetic and potential energy functions $E_{1}$ and $E_{2}$, respectively. The maximum possible peak value of $E_{1}$ produces our approximation of wave amplitude, (30), after noting that $E_{2}\left(\ln I_{\max }\right)=\beta I_{\max }$ when $z=0 . W e$ envision a particle moving in the positive $f$ direction ( $z$ decreasing from $+\infty$ ) along $E_{2}$ in (c) starting at point $\mathrm{A}$. When the particle reaches point $\mathrm{B}$, the outbreak peaks and the particle reverses direction, heading back toward point $\mathrm{C}$. Corresponding points are labeled on the invasion wave profile in (a) and on $E_{1}$ in (b).

Copyright (c) by SIAM. Unauthorized reproduction of this article is prohibited. 


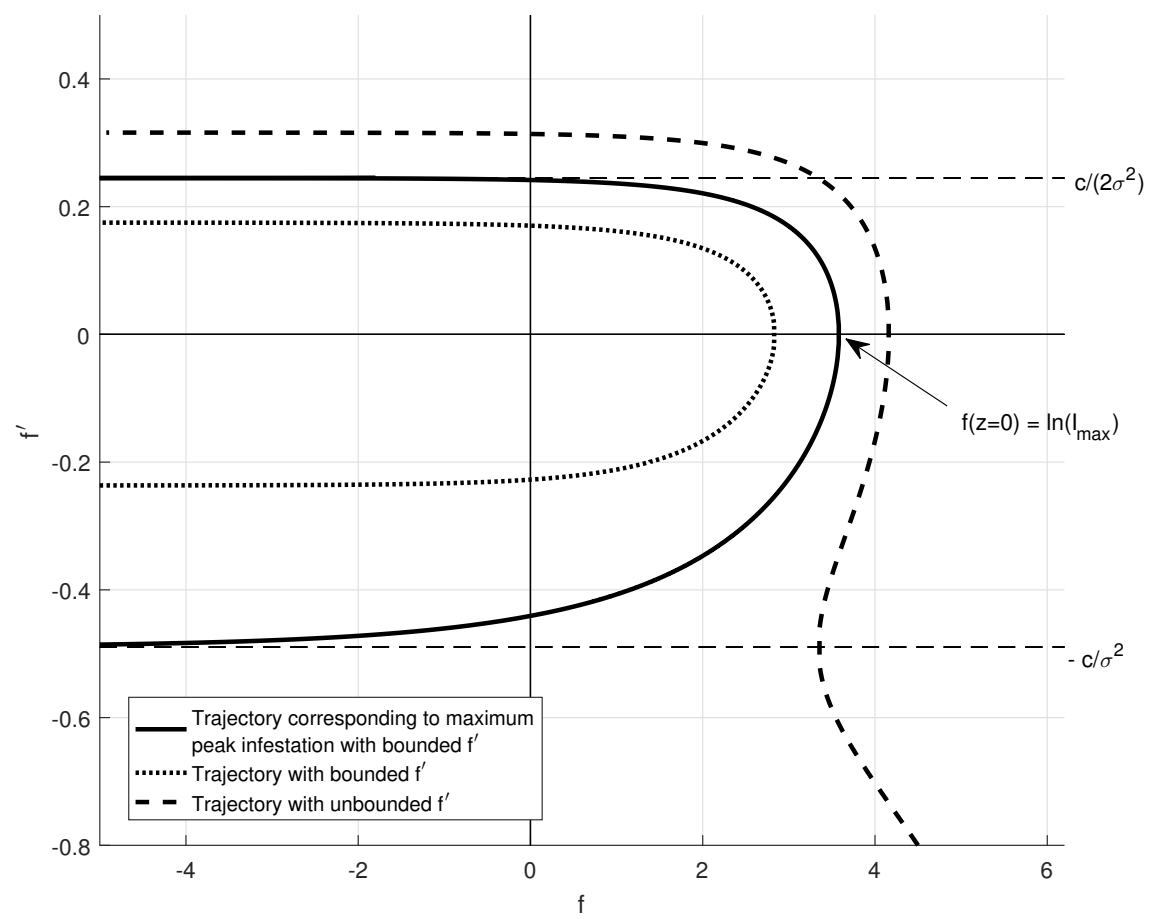

FIG. 5. Phase plane of (28). We chose the trajectory corresponding to the maximum value of $E_{1}$ for bounded $f^{\prime}$ (largest feasible amplitude of $I(x)$ ) to derive a worst case scenario approximation of $I_{\max }$. Asymptotes of this trajectory correspond to our exponential shape parameter values that describe the left and right tails of the invasion wave. Values of $f^{\prime}$ inside the interval $\left(\frac{-c}{\sigma^{2}}, \frac{c}{2 \sigma^{2}}\right)$ generate solutions with bounded $f^{\prime}$ while values outside the interval generate unrealistic (unbounded $\left.f^{\prime}\right)$ solutions.

The accuracy of the analytic approximations of the shape of an invading wave, (30), (31), and (32), is illustrated in Figure 6. To construct an approximation of the entire wave profile, we numerically integrate (28) using Euler's method (Figure 6). The slopes of the right tails of $\ln I=f$ from simulation and approximation approach the same value, $\frac{-c}{\sigma^{2}}$ as $x \rightarrow \infty$ indicating that (31) is a good approximation of the shape of the invading wave tail.

After substituting the wave speed, $c^{*}$, from (18), the approximation of the exponential decay rate of the advancing wave's leading edge in (31), $\frac{-\sqrt{2 \ln R}}{\sigma}$, agrees exactly with the wave shape parameter prediction (19). This is no surprise since (31) is based on the the maximum feasible value of $E_{1}$, and the method employed in the derivation of (19) provides a maximum wave speed using dominant eigenvalues and wave growth rates.

Using the approximation of invasion speed, $c^{*}$, from (18) in (30), we obtain a prediction of wavefront amplitude in terms of the net reproductive rate of MPBs, $R$, and MPBs host searching parameter, $\beta$,

$$
I_{\max }=\frac{2(\ln R)^{2}}{3 \beta} .
$$

5. Approximations versus model simulation. Figure 7 compares predicted invasion wavefront speeds for varying MPB population growth rate, $R$, using (18) with 

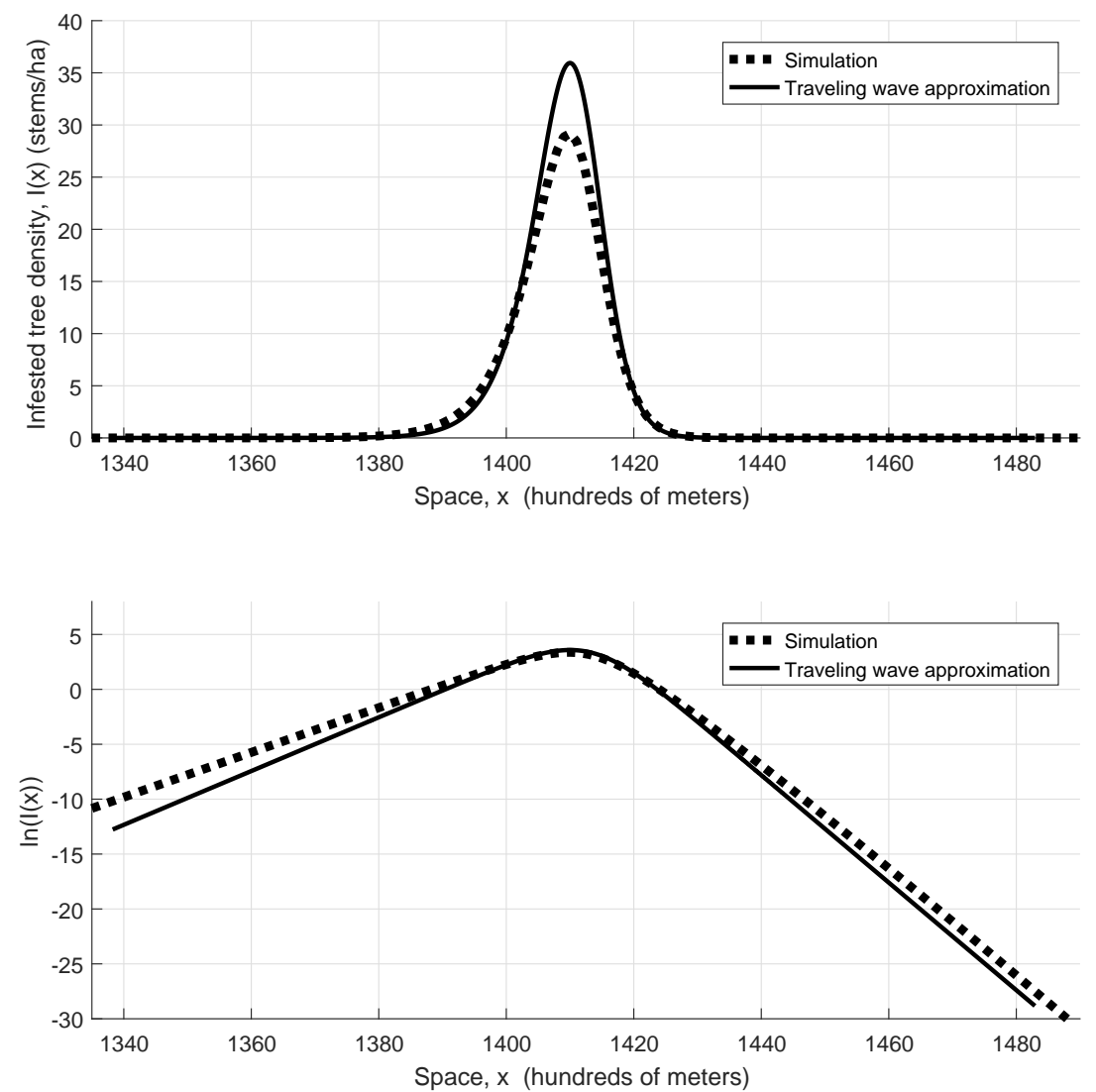

FIG. 6. Comparison of the numerical solution of (28) with the actual wave profile generated by model simulation. The slopes of the right tails of $\ln I=f$ from simulation and approximation approach the same value, $\frac{-c}{\sigma^{2}}$, as $x \rightarrow \infty$ meaning (31) is a good approximation of the shape of the invading wave tail. There is only a small error in comparing the slopes of the left tails of $\ln I$ from simulation and approximation (32).

speeds from simulation of the model ((1), (2), (4), and (7)). With nominal parameter values (Table 1), we predict an invasion speed of $315 \mathrm{~m} / \mathrm{yr}$. There is a less than $1 \%$ error between this prediction and the speed from simulation $(312 \mathrm{~m} / \mathrm{yr})$. Also note that wave speed is increasing with intrinsic beetle growth rate.

Figure 8 compares our prediction of invasion wave amplitude (33) with peaks from model simulation for varying MPB reproductive rate $R$. For nominal parameter values, maximum infestation from simulation is 29.2 stems/ha. Approximation (33) predicts peak infestation at 35.9 stems/ha. The $23 \%$ relative error is due to truncating terms that may not be negligible in the series expansion (21) since $\sigma^{2}$ is not necessarily small. In fact, since $\sigma$ and $x$ are the only variables with spatial units, nondimensionalization results in $\sigma=1$. Even so, the approximation does a relatively good job tracking the general trend of positive association between outbreak severity and insect population growth rate. Furthermore, there is only a $14.8 \%$ mean relative error over all (reasonable) $R$ values between 1.1 and 5.0. Figure 9 compares our prediction of wave amplitude (33) with maximum infestation from model simulation 


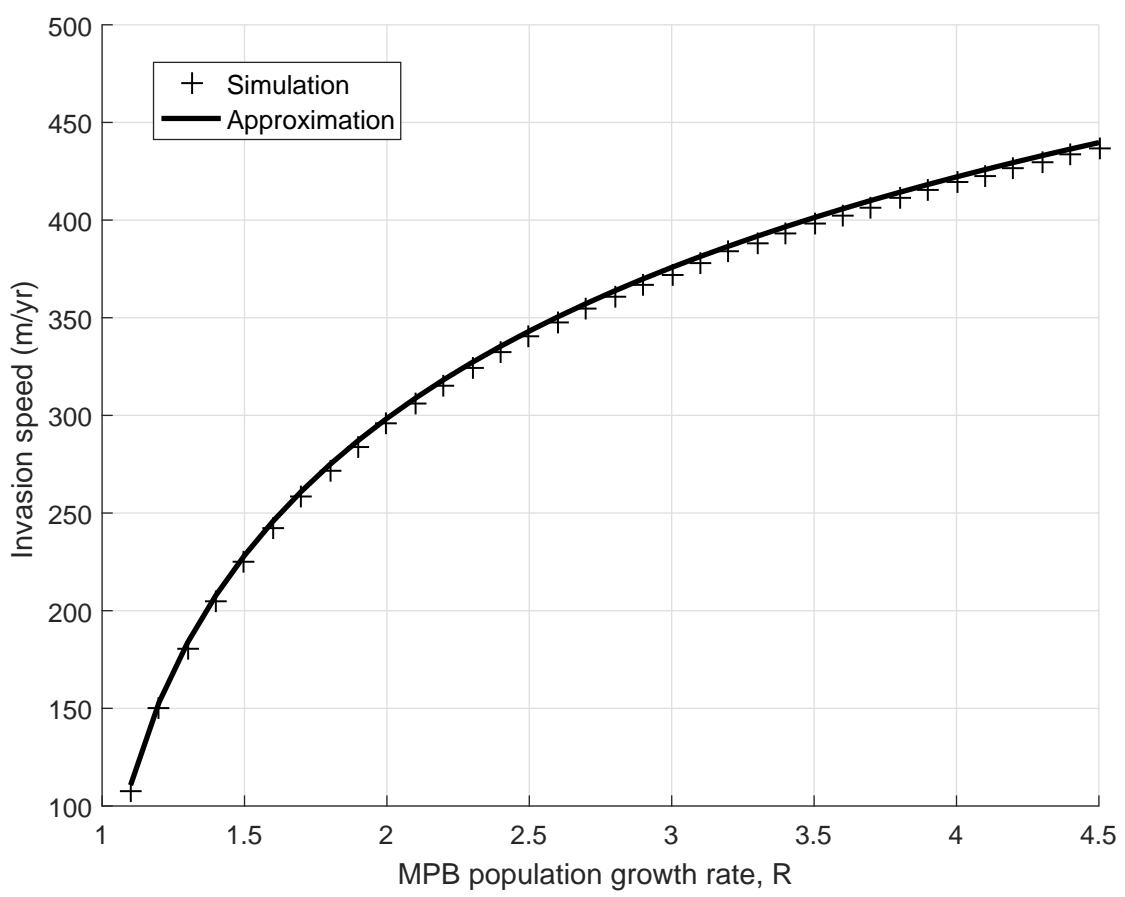

FIG. 7. Comparison of predicted wave speeds for varying $R$ using (18) with speeds from simulation. Simulation speeds were obtained by taking the average distance traveled by the leading wave's peak per year after a sufficient number of generations had elapsed (to estimate asymptotic wave speed). With the parameter values given in Table 1 , we predict $315 \mathrm{~m} /$ year for the invasion speed which is very close to the estimated speed from simulation, $312 \mathrm{~m} /$ year.

for varying MPB host searching failure rate $\beta$. Approximated peak values follow simulation peaks closely and it is clear that outbreak severity diminishes as search inefficiency, $\beta$, increases.

6. Discussion and conclusion. In this paper we present a forest insect outbreak model that emulates sedentary host population demographics. The model captures the temporal dynamics of an outbreak-recovery cycle by incorporating latency classes of nonsusceptible juveniles to account for size requirements for attacksusceptible hosts. Insect dispersal was modeled using a Gaussian dispersal kernel which is consistent with the commonly used diffusion model for insect dispersal.

Initializing the model with a small infestation near the origin generates a train of solitary waves of infestation that propagate through a medium of hosts in space. The rate at which insects disperse, as well as their net reproductive rate, determines the speed of these periodic waves which we predict analytically. From the IDE model for infested hosts, we derive an approximating PDE from which the traveling wave ODE predicts the shape and amplitude of an invading outbreak wave profile as functions of wave speed and, subsequently, pest growth rate and search efficiency.

At any given point in space, a passing infestation wave appears as a local outbreak in time. Duncan et al. [15] provide a detailed temporal analysis of local outbreaks generated by a similar host demographic model sans explicit insect dispersal. In fact, were there no dispersal component in our model, i.e., $\sigma^{2}=0$, then the PDE in (27) 


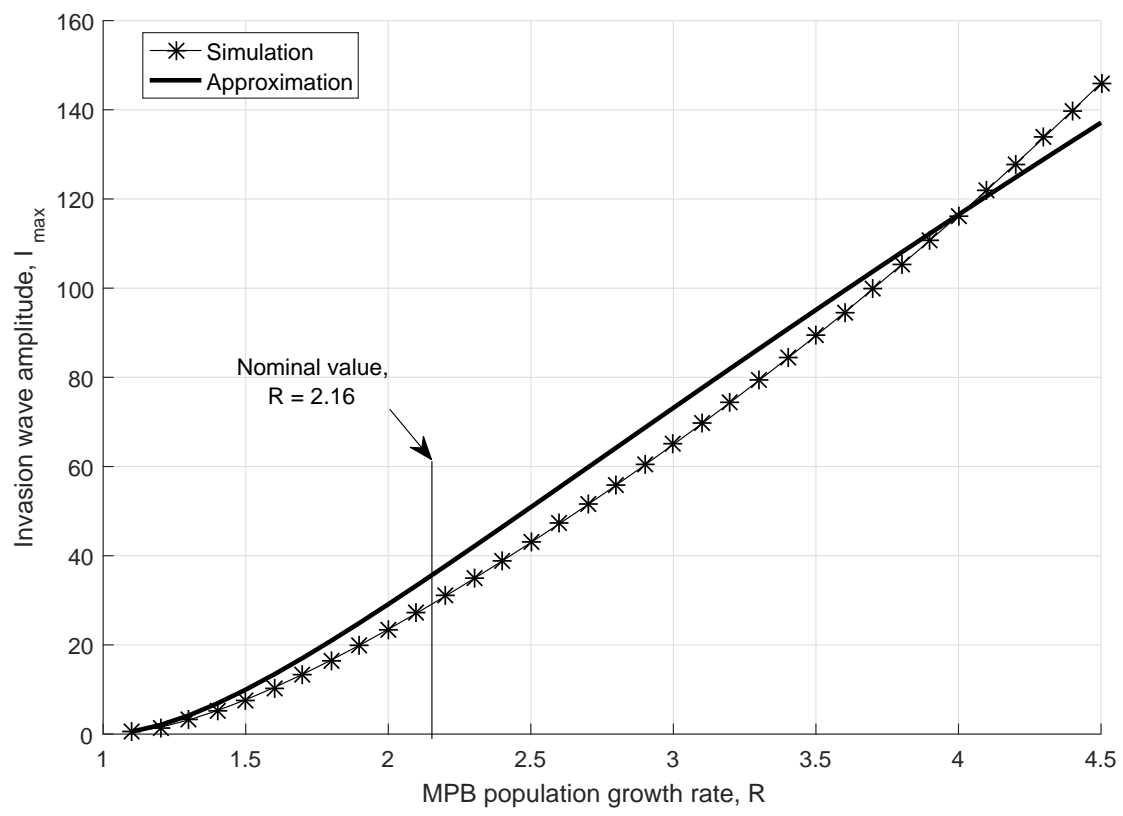

FIG. 8. Comparison of predicted invasion wave amplitude (peak infestation), $I_{\max }$, using (33) with actual peaks from simulation. Approximated peaks follow simulation peaks quite well and illuminate the positive association between outbreak severity and pest growth rate.

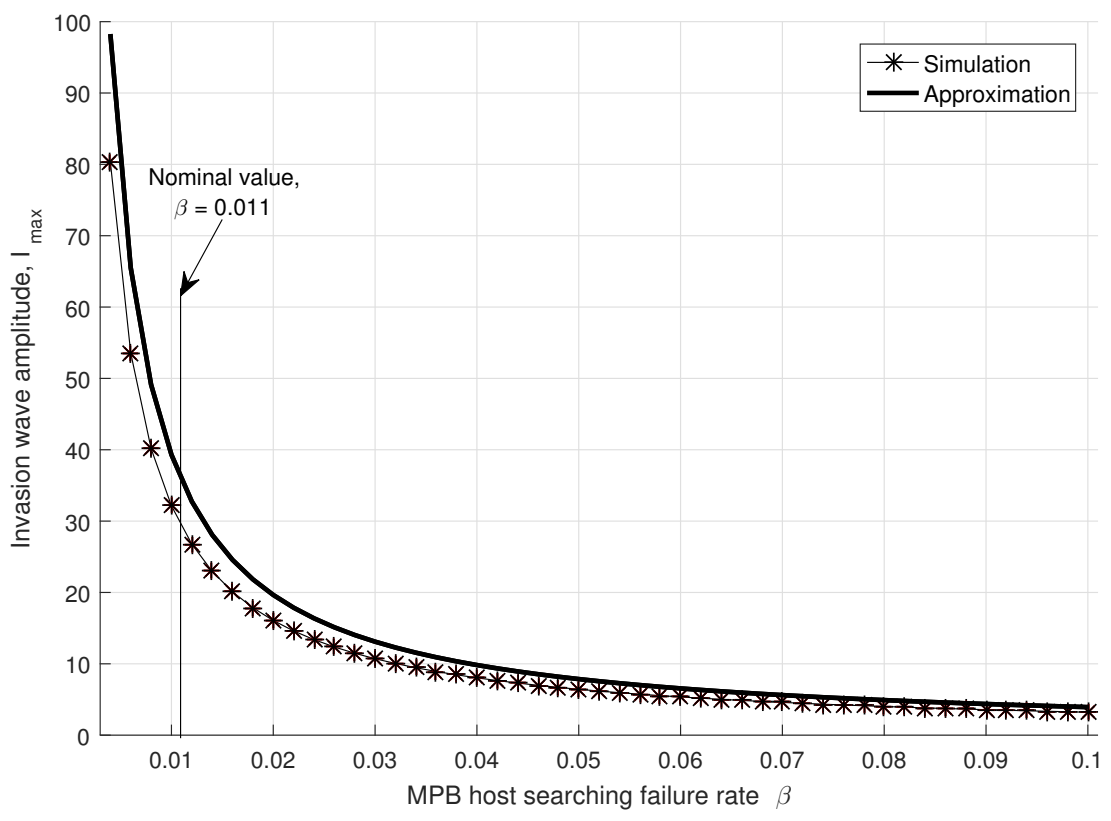

FIG. 9. Comparison of predicted invasion wave amplitude using (33) with actual peak infestation, $I_{\max }$, from simulation. Approximated peak values follow simulation peaks closely as outbreak severity diminishes with increasing search inefficiency, $\beta$.

Copyright (C) by SIAM. Unauthorized reproduction of this article is prohibited. 


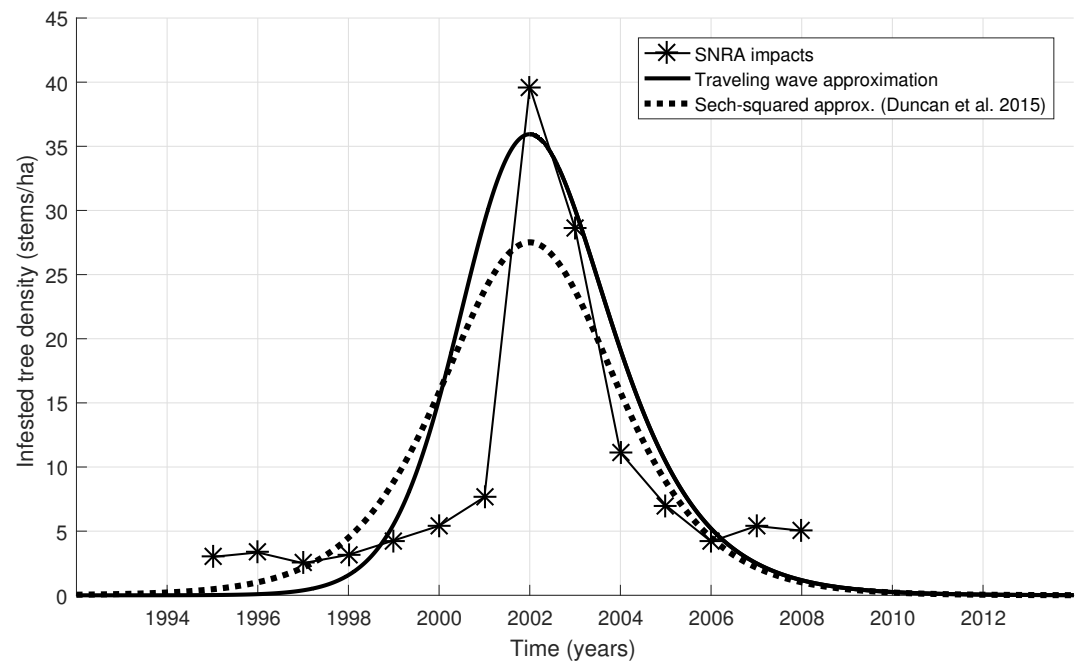

FIG. 10. Comparison of the temporal dynamics of the invasion wave (as it passes over a particular point) with the sech-squared approximation of Duncan et al. (2015) [15] as well as with infestation data from the SNRA outbreak. The traveling wave approximation is the numerical solution of (28).

would reduce to the ODE, $w_{t t}=-\beta e^{w}$, whose solution after noting that $I=e^{w}$ is

$$
I(t)=I_{\max } \operatorname{sech}^{2}\left(\sqrt{\frac{\beta I_{\max }}{2}} t\right),
$$

which is the explicit approximation of the outbreak curve in time derived in Duncan et al. [15] under the assumption that $I$ attains its maximum value, $I_{\max }$, when $t=0$.

In order to better assess the accuracy of our outbreak severity prediction (33), we compare the approximation to infestation data taken during a recent outbreak in the SNRA of central Idaho. Field observations of MPB infestation were derived from aerial detection surveys conducted by the US Forest Service whereby observers in fixed-wing aircraft measured the area impacted by MPB infestation with $30 \mathrm{~m}$ resolution; see Powell and Bentz [29] for a detailed explanation of these data. For comparison with approximation (33), we calculate the average (per ha) infestation across the SNRA each year between 1995 and (Figure 10).

A passing wave of infestation at a single point leads to a skewed outbreak wave profile with respect to time while the sech-squared function of Duncan et al. [15] is symmetric (Figure 10). Our prediction of outbreak severity is in relatively close agreement with the corresponding approximation made by Duncan et al. [15]. However, our prediction (33) is closer to the observed value from the SNRA outbreak and also captures the skewness of the outbreak wave. It is yet an open question as to whether or not it is possible to derive an analytic approximation (analogous to the sech-squared function of Duncan et al. [15] for the nonspatial model) of our invasion wave profile.

The main results provide relatively simple analytic predictions of outbreak severity and rates of spread for a variety of forest pests based on measurable biological parameters - net reproduction rate, mean dispersal distance, and host search efficiency. It is interesting to point out that while age structure plays an important part in determining the frequency of outbreaks, it does not factor in to our approximation of 
invasion speed or peak infestation (see Duncan et al. [15] for a more detailed discussion on this) since we have assumed that natural mortality of susceptible trees is negligible during an outbreak. Thus, most of our analysis applies to any disease propagating through sedentary hosts, provided dispersal can be modeled using exponential kernels with continuous exponents. Gaussian (diffusive) dispersal is probably the most commonly used kernel for modeling dispersal, but it is not ubiquitous, and further work would be required to adapt our techniques to alternate kernels. Although Gaussiantype dispersal is a limitation, here we have provided the first analytic description of the impact of traveling waves of infestation for epidemics with discrete temporal dynamics. Many, if not most, invertebrate pests in temperate climates require year-to-year models to accurately represent life-cycle dynamics, and in these systems results described here will provide a priori estimates of spread rates and degrees of potential impact.

\section{REFERENCES}

[1] G. Abramson, V.M. Kenkre, T.L. Yates, and R.R. Parmenter, Traveling waves of infection in the Hantavirus epidemics, Bull. Math. Biol., 65 (2003), pp. 519-534.

[2] R.I. Alfaro, R. Campbell, P. Vera, B. Hawkes, and T.L. Shore, Dendroecological reconstruction of mountain pine beetle outbreaks in the Chilcotin Plateau of British Columbia, in Mountain Pine Beetle Symposium: Challenges and Solutions. T.L. Shore, J.E. Brooks, and J.E. Stone, eds., Pacific Forestry Center, Victoria, British Columbia, Canada, 2004, pp. 245-256.

[3] G. Amman and W.E. Cole, Mountain Pine Beetle Dynamics in Lodgepole Pine Forests: Part II Population Dynamics, U.S. Department of Agriculture, General Technical Report INT145, Intermountain Forest and Range Experiment Station, Ogden, UT, 1983.

[4] R.M. Anderson And R.M. May, Population biology of infectious diseases: Part I, Nature, 280 (1979), pp. 361-362.

[5] R.M. Anderson and R.M. May, The logic of vaccination, New Scientist, 96 (1982), pp. 410415.

[6] D.G. Aronson and H.F. Weinberger, Multidimensional nonlinear diffusion arising in population genetics, Adv. Math., 30 (1978), pp. 33-76.

[7] B.H. Aukema, A.L. Carroll, Y. Zheng, J. Zhu, K.F. Raffa, R.D. Moore, K. Stahl, and S.W. TAYLOR, Movement of outbreak populations of mountain pine beetle: Influences of spatiotemporal patterns and climate, Ecogeography, 31 (2008), pp. 348-358.

[8] J.N. Axelson, R.I. Alfaro, And B.C. Hawkes, Influence of fire and mountain pine beetle on the dynamics of lodgepole pine stands in British Columbia, Canada, For. Ecol. Manag., 257 (2009), pp. 1874-1882.

[9] E. Ben-Jacob, H. Brand, G. Dee, L. Kramer, and J.S. Langer, Pattern propagation in nonlinear dissipative systems, Phys. D, 14 (1985), pp. 348-364.

[10] A.A. Berryman, B. Dennis, K.F. Raffa, And N.C. Stenseth, Evolution of optimal group attack, with particular reference to bark beetles (Coleoptera: Scolytidae), Ecology, 66 (1985), pp. 898-903.

[11] O.N. Bjørnstad, M. Peltonen, A.M. Liebhold, and W. Baltensweiler, Waves of larch bud moth outbreaks in the European Alps, Science, 298 (2002), pp. 1020-1023.

[12] K.P. Bleiker, M.R. OBrien, G.D. Smith, and A.L. Carroll, Characterisation of attacks made by the mountain pine beetle (Coleoptera: Curculionidae) during its endemic population phase, Canad. Entomol., 146 (2014), pp. 271-284.

[13] B.A. Crabb, J.A. Powell, and B.J. Bentz, Development and Assessment of 30-m Pine Density Maps for landscape-Level Modeling of Mountain Pine Beetle Dynamics, in Res. Pa RMRS-RP-96WWW. U.S. Department of Agriculture, Forest Service, Rocky Mountain Research Station, Fort Collins, CO, 2012.

[14] R.B. Dingle, (1973) Asymptotic Expansions: Their Derivation and Interpretation, Academic, London, 1973.

[15] J.P. Duncan, J.A. Powell, L.F. Gordillo, and J. Eason, A model for mountain pine beetle outbreaks in an age-structured forest: Predicting severity and outbreak-recovery cycle period, Bull. Math. Biol., 77 (2015), pp. 1-29.

[16] A. ERdelyi, Asymptotic Expansions, Dover, New York, 1957.

[17] R.A. Fisher, The wave of advance of advantageous genes, Ann. Eugenics, 7 (1937), pp. 355369.

Copyright $@$ by SIAM. Unauthorized reproduction of this article is prohibited. 
[18] J. Heavilin and J. Powell, A novel method for fitting spatio-temporal models to data, with applications to the dynamics of mountain pine beetle, Natur. Res. Model., 21 (2008), pp. 489524.

[19] J. Heavilin, J.A. Powell, And J.A. Logan, Development and parametrization of a model for bark beetle disturbance in lodgepole forest, in Plant Disturbance Ecology, K. Miyanishi and E. Johnson, ed., Academic, NY, 2007, pp. 527-553.

[20] H.W. Нетнсоте, The mathematics of infectious diseases, SIAM Rev., 42 (2000), pp. 599-653.

[21] H.W. Нетнсоте, A thousand and one epidemic models, in Frontiers in Mathematical Biology, Springer, Berlin, 1994, pp. 504-515.

[22] C.K. Jones, T.M. Kapitula, and J.A. Powell, Nearly real fronts in a Ginzburg-Landau equation, Proc. Roy. Soc. Edinburgh, Sect. A, 116 (1990), pp. 193-206.

[23] M.J. KeELing, The effects of local spatial structure on epidemiological invasions, R. Soc. Lond. Proc. Ser. Biol. Sci., 266 (1999), pp. 859-867.

[24] W.O. Kermack and A.G. McKendrick, A contribution to the mathematical theory of epidemics, R. Soc. Lond. Proc. Ser. A Math. Phys. Eng. Sci., 115 (1927), pp. 700-721 doi:10.1098/rspa.1927.0118.

[25] A.N. Kolmogorov, I.G. Petrovsky, And N.S. Piskunov, Investigation of the equation of diffusion combined with increasing of the substance and its application to a biology problem, Bull. Moscow State Univ. Ser. A Math. Mech., 1 (1937), pp. 1-25.

[26] M. Кot, M.A. Lewis, And P. van den Driessche, Dispersal data and the spread of invading organisms, Ecology, 77 (1996), pp. 2027-2042.

[27] J.A. Logan And J.A. Powell, Ghost forests, global warming, and the mountain pine beetle, Amer. Entomol., 47 (2001), pp. 160-173.

[28] J.A. Logan, J. RÉgnière, And J.A. Powell, Assessing the impacts of global warming on forest pest dynamics, Front. Ecol. Environ., 1 (2003), pp. 130-137.

[29] J.A. Powell AND B.J. Bentz, Connecting phenological predictions with population growth rates for an outbreak insect, Landscape Ecol., 24 (2009), pp. 657-672.

[30] J.A. Powell And B.J. Bentz, Phenology and density-dependent dispersal predict patterns of mountain pine beetle (Dendroctonus ponderosae) impact, Ecol. Model, 273 (2014), pp. 173185.

[31] S. RILEY, Large-scale spatial-transmission models of infectious disease, Science, 316 (2007), pp. 1298-1301.

[32] W.H. Romme, D.H. Knight, And J.B. YavitT, Mountain pine beetle outbreaks in the Rocky Mountains: Regulators of primary productivity?, Amer. Natur., 127 (1986), pp. 484-494.

[33] S. RuAn AND D. XIAO, Stability of steady states and existence of traveling waves in a vectordisease model, Proc. Roy. Soc. Edinburgh, Sect. A, 134 (2004), pp. 991-1011.

[34] J. Schmid And G. Amman, Dendroctonus beetles and old-growth forests in the Rockies, in OldGrowth Forest in the Southwest and Rocky Mountain Regions, M.R. Kaufmann, W.H. Moir, and W.H. Bassett, Tech. eds., USDA Forest Service General technical report RMGTR-213, Rocky Mountain Research Station, Fort Collins, CO, 1992, pp. 51-59.

[35] J.A. Sherratt, On the evolution of periodic plane waves in reaction-diffusion systems of $\lambda-\omega$ type, SIAM J. Appl. Math., 54 (1994), pp. 1374-1385.

[36] J.A. SHERRATT, Invading wave fronts and their oscillatory wakes are linked by a modulated traveling phase resetting wave, Phys. D, 117 (1998), pp. 145-166.

[37] F. van den Bosch, J.A. Metz, and O. Diekmann, The velocity of spatial population expansion, J. Math. Biol., 28 (1990), pp. 529-565.

[38] D.L. Wood, The role of pheromones, kairomones, and allomones in the host selection and colonization behavior of bark beetles, Ann. Rev. Entomol., 27 (1982), pp. 411-446.

Copyright $@$ by SIAM. Unauthorized reproduction of this article is prohibited. 